\title{
On the equivalence between some kinds of implicit and explicit iterations with applications
}

Shih-sen Chang ${ }^{1}$, Jong Kyu Kim² ${ }^{2 *}$ HW Joseph Lee ${ }^{3}$ and Chi Kin Chan ${ }^{3}$

* Correspondence:

jongkyuk@kyungnam.ac.kr

${ }^{2}$ Department of Mathematics

Education, Kyungnam University,

Masan, Kyungnam 631-701, South

Korea

Full list of author information is

available at the end of the article

\section{Abstract}

The purpose of this article is to study the equivalence between some kinds of implicit and explicit iterative approximations. As applications, we utilize our results to study the approximation problems arising in nonexpansive semigroup, variational inclusions and equilibrium problem.

Keywords: Reich's convergence theorem, Xu's convergence theorem, Browder's property, Halpern's property, fixed point, Moudafi's viscosity approximation, nonexpansive mapping, Suzuki's type strong convergence theorem, weak contractive mapping.

\section{Introduction}

Definition 1.1 Let $(X, d)$ be a metric space and let $S: X \rightarrow X$ be a mapping. $S$ is said to be $\psi$-weakly contractive (weak contraction, for short) [1], if there exists a continuous and strictly increasing function $\psi:[0,+\infty) \rightarrow[0,+\infty)$ with $\psi(t)>0, \forall t \in(0,+\infty)$ and $\psi(0)=0$ such that

$$
d(S x, S y) \leq d(x, y)-\psi(d(x, y)), \quad \forall x, y \in X
$$

From Definition 1.1, it is easy to see that if $\psi(t)=(1-h) t, h \in(0,1)$, then $S$ is a contractive mapping with the contractive constant $h$.

In 2001, Rhoades [2] proved the following interesting fixed point theorem:

Theorem 1.2 (Rhoades [2]) Let $(X, d)$ be a complete metric space and let $T: X \rightarrow X$ be a weakly contractive mapping. Then $T$ has a unique fixed point in $X$. Moreover, for any given $x \in X$, the iterative sequence $\left\{T^{n} x\right\}$ converges strongly to this fixed point.

This theorem is one of the generalization of Banach contraction Theorem.

Let $E$ be a Banach space and let $C$ be a nonempty, closed and convex subset of $E$. A mapping $T: C \rightarrow C$ is said to be nonexpansive, if

$$
\|T x-T y\| \leq\|x-y\|, \quad \text { for all } x, y \in C .
$$

Denote by $F(T)$ the fixed point set of $T$. It is well-known that if $E$ is uniformly smooth $C$ is bounded and $T: C \rightarrow C$ is nonexpansive then $F(T)$ is nonempty (see, Göhde [3]).

Recently, many convergence theorems to fixed points of nonexpansive mappings have been established. 
In 1967, Browder [4] and Halpern [5] proved some strong convergence theorems in Hilbert spaces for implicit and explicit iteration, respectively. Later these theorems have been extended in several directions. In 1980, Reich [6] proved the following:

Theorem 1.3 (Reich [6]) Let $E$ be a uniformly smooth Banach space, $C$ be a bounded, closed and convex subset of $E$ and let $T: C \rightarrow C$ be a nonexpansive mapping. Fix $u \in C$ and define a net $\left\{y_{t}\right\}$ in $C$ by

$$
y_{t}=t u+(1-t) T y_{t}, \quad t \in(0,1) .
$$

Then $\left\{y_{t}\right\}$ converges strongly to $P u$ as $t \rightarrow 0+$, where $P$ is the unique sunny nonexpansive retraction from $C$ onto $F(T)$. (The relative definitions are given in Section 2.)

In the sequel, this theorem is called Reich's convergence theorem.

In $2004 \mathrm{Xu}$ [7] proved the following

Theorem 1.4 (Xu [7]) Let $E$ be a uniformly smooth Banach space and let $C$ be a bounded, closed and convex subset of $E$. Let $T: C \rightarrow C$ be a nonexpansive mapping with $F(T) \neq \varnothing, P$ be the unique sunny nonexpansive retraction from $C$ onto $F(T)$ and let $f: C \rightarrow C$ be a contractive mapping with a contractive constant $h \in(0,1)$. Let $\left\{x_{t}\right\}$ be the net in $C$ which is defined by

$$
x_{t}=t f\left(x_{t}\right)+(1-t) T x_{t}, \quad t \in(0,1) .
$$

Then as $t \rightarrow 0+,\left\{x_{t}\right\}$ converges strongly to the unique point $z \in C$ satisfying $P \nabla f(z)$ $=z$.

On the other hand, in 2002, Xu proved the following.

Theorem 1.5 (Xu $[8,9])$ Let $E, C, T, P$ be as in Theorem 1.3. Let $\left\{y_{n}\right\}$ be the sequence in $C$ defined by $y_{1} \in C$ and

$$
y_{n_{+1}}=\alpha_{n} u+\left(1-\alpha_{n}\right) T y_{n}, \quad \forall n \geq 1,
$$

where $\left\{\alpha_{n}\right\}$ is a sequence in $(0,1)$ which satisfies the following conditions:

(C1) $\lim _{n \rightarrow \infty} \alpha_{n}=0$;

(C2) $\sum_{n=1}^{\infty} \alpha_{n}=\infty$;

(C3) $\lim _{n \rightarrow \infty} \frac{\alpha_{n+1}}{\alpha_{n}}=1$.

Then $\left\{y_{n}\right\}$ converges strongly to $\mathrm{Pu}$.

In the sequel, this theorem is called Xu's convergence theorem.

Moreover, in 2000, Moudafi [10] introduced the concept of viscosity approximation and proved the following result

Theorem 1.6 (Moudafi [10]) Let $H$ be a real Hilbert space, $C$ be a nonempty closed convex subset of $H, T: C \rightarrow C$ be a nonexpansive mapping with $F(T) \neq \varnothing$ and $f: C \rightarrow$ $C$ be a contractive mapping. Let $\left\{x_{n}\right\}$ be the sequence defined by:

$$
x_{n+1}=\alpha_{n} f\left(x_{n}\right)+\left(1-\alpha_{n}\right) T x_{n}, \quad \forall n \geq 1 .
$$

Then $\left\{x_{n}\right\}$ converge strongly to a fixed point of $T$ in Hilbert space $H$.

In the sequel, this theorem is called Moudafi's viscosity convergence theorem.

Moudafi's viscosity convergence theorem is very important, because it can be applied to convex optimization, linear programming, monotone inclusions and elliptic differential equations (see [11]). 
In 2004, Xu [7] extended Moudafi's results to a uniformly smooth Banach space. Song and Chen [12-14] also obtained some strong convergence theorems about viscosity approximations in more general Banach space. More recently, Suzuki [11] and Petrusel and Yao [15] replace a contraction $f$ in (1.6) by a Meir-Keeler contraction [16] and a generalized contraction [15], respectively, to approximate a common fixed point for an infinite family of nonexpansive mappings.

The purpose of this article is to study the equivalence between some kinds of implicit and explicit iterative approximations approximations. As applications, we utilize our results to study some approximation problems arising in nonexpansive semigroup, variational inclusions and equilibrium problems.

\section{Preliminaries}

In this section, we give some preliminaries which will be needed in proving our main results.

Throughout this article, we assume that $E$ is a real Banach space, $E^{*}$ is the dual of $E$. The space $E$ is said to be smooth or said to have a Gâteaux differentiable norm, if the limit $\lim _{t \rightarrow 0} \frac{\|x+t y\|-\|x\|}{t}$ exists for each $x, y \in U=\{x \in E:\|x\|=1\}$. The space $E$ is said to have a uniformly Gâteaux differentiable norm, if for each $y \in U$, the limit is attained uniformly in $x \in U$. The space $E$ is said to be uniformly smooth or said to have a uniformly Fréchet differentiable norm, if the limit is attained uniformly in $x, y \in U$.

Let $C$ and $K$ be subsets of $E$. Recall that A mapping $P: C \rightarrow K$ is called sunny, if $P(P x$ $+t(x-P x))=P x$ for any $x \in C$ with $P x+t(x-P x) \in C$ and $t \geq 0$.

Let $C$ be a convex subset of $E$ and $K$ be a subset of $C$. A mapping $P: C \rightarrow K$ is called retraction from $C$ onto $K$, if $P^{2}=P$.

Lemma 2.1 (Reich [17]) Let $E$ be a smooth Banach space, and $J: E \rightarrow E^{*}$ be the normalized duality mapping. Let $C$ be a convex subset of $E, K$ be a subset of $C$ and let $P$ be a retraction from $C$ onto $K$. Then the following are equivalent:

(1) $\langle x-P x, J(P x-y)\rangle \geq 0, \forall x \in C$ and $y \in K$;

(2) $P$ is sunny and nonexpansive.

Hence there is at most one sunny nonexpansive retraction from $C$ onto $K$.

Remark 2.2 We note that if $E$ is a real Hilbert space and $K$ is a closed and convex subset of $C$, then the metric projection and the sunny nonexpansive retraction from $C$ onto $K$ coincide, i.e., when $T: C \rightarrow C$ is a nonexpansive mapping, the sunny nonexpansive retraction from $C$ onto $F(T)$ is the metric projection.

Proposition 2.3 (Suzuki [11]) Let $E$ be a smooth Banach space and let $C$ be a closed and convex subset of $E$. Let $K$ be a subset of $C$ and let $P$ be the unique sunny nonexpansive retraction from $C$ onto $K$. Let $f: C \rightarrow C$ be a mapping and $z \in K$ be a point. Then the following statements are equivalent:

(1) $z$ is a fixed point of $P \nabla f$;

(2) $z$ is a solution to the following variational inequality:

$$
\langle f z-z, J(z-y)\rangle \geq 0, \quad \forall v \in K .
$$

Proposition 2.4 (Song [14]) Let $E$ be a Banach space and let $C$ be a convex subset of $E$. Let $T: C \rightarrow C$ be a nonexpansive mapping and let $g: C \rightarrow C$ be a weakly contractive mapping. Then the following conclusions hold:

(1) $T \nabla g$ is weakly contractive; 
(2) for each $t \in(0,1)$, the mapping $x \propto \operatorname{tg}(x)+(1-t) T x$ is weakly contractive and the net $\left\{x_{t}\right\}$ defined by

$$
x_{t}=\operatorname{tg}\left(x_{t}\right)+(1-t) \operatorname{Tx} x_{t}
$$

is well-defined.

Lemma 2.5 (Alber and Guerre-Delabriere [1]) Let $\left\{\lambda_{n}\right\}$ and $\left\{\beta_{n}\right\}$ be two sequences of nonnegative real numbers and let $\left\{\alpha_{n}\right\}$ be a sequence of positive numbers satisfying the conditions $\sum_{n=1}^{\infty} \alpha_{n}=\infty$ and $\lim _{n \rightarrow \infty} \frac{\beta_{n}}{\alpha_{n}}=0$. Let the recursive inequality

$$
\lambda_{n+1} \leq \lambda_{n}-\alpha_{n} \psi\left(\lambda_{n}\right)+\beta_{n}, \quad \forall n \geq 1
$$

be given where $\psi:[0,+\infty) \rightarrow[0,+\infty)$ is a continuous and strictly increasing function with $\psi(0)=0$. Then $\left\{\lambda_{n}\right\}$ converges to 0 as $n \rightarrow \infty$.

\section{Equivalence between some implicit and explicit iterative schemes}

We first give the following result.

Theorem 3.1 Let $E$ be a uniformly smooth Banach space and let $C$ be a bounded, closed and convex subset of $E$. Let $T: C \rightarrow C$ be a nonexpansive mapping with $F(T) \neq$ $\varnothing, P$ be the unique sunny nonexpansive retraction from $C$ onto $F(T)$ and let $g: C \rightarrow C$ be a $\psi$-weakly contractive mapping. Let $\left\{x_{t}\right\}$ be the net in $C$ defined by

$$
x_{t}=\operatorname{tg}\left(x_{t}\right)+(1-t) T x_{t}, \quad t \in(0,1) .
$$

Then $\left\{x_{t}\right\}$ converges strongly to the unique point $z \in C$ satisfying $P \nabla g(z)=z$ as $t \rightarrow$ $0+$.

Proof. Since $P$ is nonexpansive and $g$ is $\psi$-weakly contractive, by Proposition 2.4 (1), $P \nabla g: C \rightarrow C$ is $\psi$-weakly contractive. From Theorem 1.2 , there exists a unique $z \in C$ such that $z=P \nabla g(z)$.

Now we define a net $\left\{y_{t}\right\}$ as follows:

$$
y_{t}=\operatorname{tg}(z)+(1-t) T y_{t}, \quad t \in(0,1) .
$$

By virtue of Theorem 1.3, $y_{t} \rightarrow P \nabla g(z)=z$, as $t \rightarrow 0+$. Moreover, for each $t \in(0,1)$, we have

$$
\begin{aligned}
\left\|x_{t}-y_{t}\right\| \leq & t\left\|g\left(x_{t}\right)-g\left(y_{t}\right)+g\left(y_{t}\right)-g(z)\right\|+(1-t)\left\|T x_{t}-T y_{t}\right\| \\
\leq & t\left\{\left\|x_{t}-y_{t}\right\|-\psi\left(\left\|x_{t}-y_{t}\right\|\right)\right\} \\
& +t\left\{\left\|y_{t}-z\right\|-\psi\left(\left\|y_{t}-z\right\|\right)\right\}+(1-t)\left\|x_{t}-y_{t}\right\| \\
= & \left\|x_{t}-y_{t}\right\|-t \psi\left(\left\|x_{t}-y_{t}\right\|\right)+t\left\{\left\|y_{t}-z\right\|-\psi\left(\left\|y_{t}-z\right\|\right)\right\} .
\end{aligned}
$$

This implies that

$$
\psi\left(\left\|x_{t}-y_{t}\right\|\right)+\psi\left(\left\|y_{t}-z\right\|\right) \leq\left\|y_{t}-z\right\| \rightarrow 0 \text { as } t \rightarrow 0+.
$$

Since $\psi$ is continuous and strictly increasing, we have

$$
\left\|x_{t}-y_{t}\right\| \rightarrow 0,\left\|y_{t}-z\right\| \rightarrow 0 \quad \text { as } t \rightarrow 0+.
$$

Therefore we have

$$
\left\|x_{t}-z\right\| \leq\left\|x_{t}-y_{t}\right\|+\left\|y_{t}-z\right\| \rightarrow 0, \quad \text { as } t \rightarrow 0+.
$$

This completes the proof of Theorem 3.1. 
Theorem 3.2 Let E, C, T, P, $g$ be the same as in Theorem 3.1. Then Theorems 1.3, 1.4 , and 3.1 are equivalent.

Proof. Theorem $1.3 \Rightarrow$ Theorem 3.1 has been proved in Theorem 3.1. Now we proved that Theorem $3.1 \Rightarrow$ Theorem $1.4 \Rightarrow$ Theorem 1.3.

In fact, in Theorem 3.1 take $g: C \rightarrow C$ to be a contractive mapping, then the conclusion of Theorem 1.4 can be obtained from Theorem 3.1. As well as in Theorem 1.4 take $f$ as a constant valued mapping, then the conclusion Theorem 1.3 can be obtained from Theorem 1.4 immediately. This completes the proof of Theorem 3.2.

Remark 3.3 From Theorems 3.1 and 3.2, it follows that the convergence of the implicit iterative sequences defined by (1.3), (1.4), and (3.1) are equivalent.

Next we consider the equivalence between some explicit iterative sequences In 2004, $\mathrm{Xu}$ [7] proved the following result:

Theorem 3.4 (Xu [7]) Let $E$ be a uniformly smooth Banach space and let $C$ be a bounded, closed and convex subset of $E$. Let $T: C \rightarrow C$ be a nonexpansive mapping with $F(T) \neq \varnothing, P$ be the unique sunny nonexpansive retraction from $C$ onto $F(T)$ and let $f: C \rightarrow C$ be a contractive mapping. Let $\left\{x_{n}\right\}$ be the sequence in $C$ which is defined by

$$
x_{n+1}=\alpha_{n} f\left(x_{n}\right)+\left(1-\alpha_{n}\right) T x_{n}, \quad \forall n \geq 1,
$$

where $\left\{\alpha_{n}\right\}$ is a sequence in $(0,1)$ satisfying the conditions $(\mathrm{C} 1),(\mathrm{C} 2)$ and $(\mathrm{C} 3)$ in Theorem 1.5. Then $\left\{x_{n}\right\}$ converges strongly to the unique point $z \in C$ satisfying $P \nabla f$ $(z)=z$.

It is easy to see that Theorem 3.4 is a generalization of Moudafi's Theorem 1.6.

Now we give the following result.

Theorem 3.5 Let $E, C, T, P$ be the same as in Theorem 3.4. Let $g: C \rightarrow C$ be a $\psi$-weakly contractive mapping. Let $\left\{x_{n}\right\}$ be the sequence in $C$ which is defined by

$$
x_{n+1}=a_{n} g\left(x_{n}\right)+\left(1-a_{n}\right) T x_{n}, \quad \forall n \geq 1,
$$

where $\left\{\alpha_{n}\right\}$ is a sequence in $(0,1)$ satisfying the conditions $(\mathrm{C} 1),(\mathrm{C} 2)$, and (C3) in Theorem 1.5. Then $\left\{x_{n}\right\}$ converges strongly to the unique point $z \in C$ satisfying $P \nabla g$ $(z)=z$;

Proof. Since $P$ is nonexpansive and $g$ is a $\psi$-weak contraction, by Proposition 2.4 (1), $P \nabla g: C \rightarrow C$ is weakly contractive. From Theorem 1.2, there exists a unique $z \in C$ such that $z=P \nabla g(z)$.

Now we define a sequence $\left\{y_{n}\right\}$ as follows:

$$
y_{n_{+1}}=\alpha_{n} g(z)+\left(1-\alpha_{n}\right) T y_{n}, \quad \forall n \geq 1 .
$$

By Theorem 1.5, $y_{n} \rightarrow P \nabla g(z)=z$, as $n \rightarrow \infty$. Moreover, for each $n \geq 1$, we have

$$
\begin{aligned}
\left\|x_{n+1}-y_{n_{+1}}\right\| \leq & \alpha_{n}\left\|g\left(x_{n}\right)-g\left(y_{n}\right)+g\left(y_{n}\right)-g(z)\right\|+\left(1-\alpha_{n}\right)\left\|T x_{n}-T y_{n}\right\| \\
\leq & \alpha_{n}\left\{\left\|x_{n}-y_{n}\right\|-\psi\left(\left\|x_{n}-y_{n}\right\|\right)\right\} \\
& +\alpha_{n}\left\{\left\|y_{n}-z\right\|-\psi\left(\left\|y_{n}-z\right\|\right)\right\}+\left(1-\alpha_{n}\right)\left\|x_{n}-y_{n}\right\| \\
= & \left\|x_{n}-y_{n}\right\|-\alpha_{n} \psi\left(\left\|x_{n}-y_{n}\right\|\right)+\alpha_{n}\left\{\left\|y_{n}-z\right\|-\psi\left(\left\|y_{n}-z\right\|\right)\right\} .
\end{aligned}
$$

Letting $\lambda_{n}=\left\|x_{n}-y_{n}\right\|, \beta_{n}=\alpha_{n}\left\{\| y_{n}-z||-\psi\left(\| y_{n}-z||\right)\right\}$ in Lemma 2.5, from the assumptions, we know that all the conditions in Lemma 2.5 are satisfied. It follows from Lemma 2.5 and (3.5) that 


$$
\lim _{n \rightarrow \infty}\left\|x_{n}-y_{n}\right\|=0
$$

Therefore we have

$$
\left\|x_{n}-z\right\| \leq\left\|x_{n}-y_{n}\right\|+\left\|y_{n}-z\right\| \rightarrow 0, \quad \text { as } n \rightarrow \infty .
$$

The conclusion of Theorem 3.5 is proved.

Theorem 3.6 Let E, C, T, P, $g$ be the same as in Theorem 3.5. Then Theorems 3.5, 3.4 , and 1.5 are equivalent.

Indeed, the conclusion that Theorem $1.5 \Rightarrow$ Theorem 3.5 has been proved in Theorem 3.5. Now we proved that Theorem $3.5 \Rightarrow$ Theorem $3.4 \Rightarrow$ Theorem 1.5. In fact, in Theorem 3.5 take $g$ : $C \rightarrow C$ to be a contractive mapping, the conclusion of Theorem 3.4 can be obtained from Theorem 3.5 immediately. Again in Theorem 3.4 take $f: C \rightarrow$ $C$ to be a constant-valued mapping, the conclusion of Theorem 1.5 can be obtained from Theorem 3.4 immediately.

Remark 3.7 From Theorem 3.5, it follows that the convergence of the explicit iterative sequences defined by (1.5), (3.4), and (3.3) are equivalent.

\section{Equivalence between some more general implicit and explicit iterative schemes}

In this section we study the equivalence between some more general implicit and explicit iterative schemes. Following Suzuki [11], we introduce the following concepts.

Definition 4.1 (1) Let $E$ be a Banach space and let $C$ be a nonempty, closed and convex subset of $E$. Let $\left\{S_{n}\right\}: C \rightarrow C$ be an infinite family of nonexpansive mappings and $\left\{\alpha_{n}\right\}$ be a sequence in $(0,1)$ satisfying the condition $(C 1)$ in Theorem 1.5. The tuple $\left(E, C,\left\{S_{n}\right\},\left\{\alpha_{n}\right\}\right)$ is said to have Browder's property, if for each $u \in C$ the implicit iterative sequence $\left\{y_{n}\right\}$ defined by

$$
y_{n}=\alpha_{n} u+\left(1-\alpha_{n}\right) S_{n} y_{n}, \quad n \geq 1,
$$

converges strongly.

(2) Let $\left\{\alpha_{n}\right\}$ be a sequence in $(0,1)$ satisfying the conditions (C1) and (C2) in Theorem 1.4 Then the tuple $\left(E, C,\left\{S_{n}\right\},\left\{\alpha_{n}\right\}\right)$ is said to have Halpern's property, if for each $u \in C$ the explicit iterative sequence $\left\{y_{n}\right\}$ defined by $y_{1} \in C$ and

$$
y_{n+1}=\alpha_{n} u+\left(1-\alpha_{n}\right) S_{n} y_{n}, \quad n \geq 1,
$$

converges strongly.

It is well known that if $E$ is a Hilbert space, $C$ is a bounded subset of $E$ and $\left\{S_{n}\right\}=\{S\}$ is a constant sequence of nonexpansive mappings, then the tuple $\left(E, C,\left\{S_{n}\right\},\left\{\frac{1}{n}\right\}\right)$ has both Browder's and Halpern's property.

Lemma 4.2 (Suzuki [11])

(1) Let $\left(E, C,\left\{S_{n}\right\},\left\{\alpha_{n}\right\}\right)$ have the "Browder's property". For each $u \in C$, let $\left\{y_{n}\right\}$ be the sequence in $C$ defined by (4.1). Denote $P u=\lim _{n \rightarrow \infty} y_{n}$, then $P$ is a nonexpansive mapping from $C$ to $C$;

(2) Let $\left(E, C,\left\{S_{n}\right\},\left\{\alpha_{n}\right\}\right)$ have the "Halpern's property". For each $u \in C$, let $\left\{y_{n}\right\}$ be the sequence in $C$ defined by (4.2). Denote $P u=\lim _{n \rightarrow \infty} y_{n}$, then the following hold:

(i) $P u$ does not depend on the initial point $y_{1} \in C$;

(ii) $P$ is a nonexpansive mapping from $C$ into $C$. 
Theorem 4.3 Let $\left(E, C,\left\{S_{n}\right\},\left\{\alpha_{n}\right\}, P\right)$ has the "Browder's property". Let $g: C \rightarrow C$ be a $\psi$-weakly contractive mapping. Define a sequence $\left\{x_{n}\right\}$ in $C$ by

$$
x_{n}=\alpha_{n} g\left(x_{n}\right)+\left(1-\alpha_{n}\right) S_{n} x_{n}, \quad n \geq 1 .
$$

Then $\left\{x_{n}\right\}$ converges strongly to the unique point $z \in C$ satisfying $P \circ g z=z$.

Proof. By Proposition 2.4, the existence and uniqueness of $\left\{x_{n}\right\}$ and $z=P \circ g z$ are assured. Define a sequence $\left\{y_{n}\right\}$ in $C$ by

$$
y_{n}=\alpha_{n} g(z)+\left(1-\alpha_{n}\right) S_{n} y_{n} \quad n \geq 1 .
$$

By the assumptions, $y_{n} \rightarrow P \circ f z$ which equals to $z$. And for each $n \geq 1$, we have

$$
\begin{aligned}
\left\|x_{n}-y_{n}\right\| \leq & \alpha_{n}\left\{\left\|g\left(x_{n}\right)-g\left(y_{n}\|+\| g\left(y_{n}\right)-g(z) \|\right\}+\left(1-\alpha_{n}\right)\right\| S_{n} x_{n}-S_{n} y_{n} \|\right. \\
\leq & \alpha_{n}\left\{\left\|x_{n}-y_{n}\right\|-\psi\left(\left\|x_{n}-y_{n}\right\|\right)\right\} \\
& +\alpha_{n}\left\{\left\|y_{n}-z\right\|-\psi\left(\left\|y_{n}-z\right\|\right)\right\}+\left(1-\alpha_{n}\right)\left\|x_{n}-y_{n}\right\| .
\end{aligned}
$$

Simplifying it, we have

$$
\psi\left(\left\|x_{n}-y_{n}\right\|+\psi\left(\left\|y_{n}-z\right\|\right) \leq\left\|y_{n}-z\right\| \rightarrow 0 \quad \text { as } n \rightarrow \infty .\right.
$$

In view of the property of $\psi$, this implies that $\lim _{n \rightarrow \infty}|| x_{n}-y_{n} \|=0$. Hence we have

$$
\left\|x_{n}-z\right\| \leq\left\|x_{n}-y_{n}\right\|+\left\|y_{n}-z\right\| \rightarrow 0 \quad \text { as } n \rightarrow \infty .
$$

This completes the proof of Theorem 4.3.

Remark 4.4 It follows from Theorem 4.3 that the implicit iterative sequences (4.1) and (4.3) are equivalent.

Theorem 4.5 Let $\left(E, C,\left\{S_{n}\right\},\left\{\alpha_{n}\right\}, P\right)$ have the "Halpern's property". Let $g: C \rightarrow C$ be a $\psi$-weakly contractive mapping. Define a sequence $\left\{x_{n}\right\}$ in $C$ by $x_{1} \in C$ and

$$
x_{n+1}=\alpha_{n} g\left(x_{n}\right)+\left(1-\alpha_{n}\right) S_{n} x_{n}, \quad n \geq 1 .
$$

Then $\left\{x_{n}\right\}$ converges strongly to the unique point $z L C$ satisfying $P \circ g z=z$.

Proof. By Proposition 2.4 (1), $P \circ g$ is a $\psi$-weak contraction from $C$ to $C$. Hence there exists a unique $z \in C$ such that $z=P \circ g z$. Now we define a sequence $\left\{y_{n}\right\}$ by

$$
y_{n+1}=\alpha_{n} g(z)+\left(1-\alpha_{n}\right) S_{n} y_{n}, \quad \geq 1 .
$$

By Lemma 4.2

$$
y_{n} \rightarrow P \circ g(z)=z .
$$

Hence we have

$$
\begin{aligned}
\left\|x_{n+1}-y_{n+1}\right\| \leq & \alpha_{n}\left\|g\left(x_{n}\right)-g\left(y_{n}\right)+g\left(y_{n}\right)-g(z)\right\|+\left(1-\alpha_{n}\right)\left\|S_{n} x_{n}-S_{n} y_{n}\right\| \\
\leq & \alpha_{n}\left\{\left\|x_{n}-y_{n}\right\|-\psi\left(\left\|x_{n}-y_{n}\right\|\right)\right\} \\
& +\alpha_{n}\left\{\left\|y_{n}-z\right\|-\psi\left(\left\|y_{n}-z\right\|\right)\right\}+\left(1-\alpha_{n}\right)\left\|x_{n}-y_{n}\right\| \\
= & \left\|x_{n}-y_{n}\right\|-\alpha_{n} \psi\left(\left\|x_{n}-y_{n}\right\|\right)+\alpha_{n}\left\{\left\|y_{n}-z\right\|-\psi\left(\left\|y_{n}-z\right\|\right)\right\} .
\end{aligned}
$$

Taking $\lambda_{n}=|| x_{n}-y_{n} \|, \beta_{n}=\alpha_{n}\left\{\| y_{n}-z||-\psi\left(\| y_{n}-z||\right)\right\}$ in Lemma 2.5 and from the assumptions, we know that all the conditions in Lemma 2.5 are satisfied, and therefore we have

$$
\lim _{n \rightarrow \infty}\left\|x_{n}-y_{n}\right\|=0,
$$


which implies that

$$
\left\|x_{n}-z\right\| \leq\left\|x_{n}-y_{n}\right\|+\left\|y_{n}-z\right\| \rightarrow 0, \quad \text { as } n \rightarrow \infty .
$$

This completes the proof of Theorem 4.5.

Remark 4.6 From the above arguments, we get that the explicit iterative sequences (4.2) and (4.4) are equivalent.

\section{Applications}

In this section, we will utilize Theorems 4.3 and 4.5 to study some approximation problems and to state several deduced theorems.

\subsection{Application to nonexpansive semigroup problems}

Theorem 5.1 Let $E$ be a uniformly convex Banach space having a weakly continuous normalized duality mapping $J: E \rightarrow E^{*}, C$ be a closed and convex subset of $E$ and let $\mathcal{T}:=\{T(t): t \geq 0\}: C \rightarrow C$ be a nonexpansive semigroup with $F(\mathcal{T}):=\bigcap_{t \geq 0} F(T(t)) \neq \emptyset$. Let $g: C \rightarrow C$ be a $\psi$-weakly contractive mapping and let $\left\{x_{n}\right\}$ be the implicit iterative sequence defined by:

$$
x_{n}=\alpha_{n} g\left(x_{n}\right)+\left(1-\alpha_{n}\right) T\left(t_{n}\right) x_{n}, \quad \forall n \geq 1,
$$

where $\left\{\alpha_{n}\right\}$ and $\left\{t_{n}\right\}$ are sequences of real numbers such that $\alpha_{n} \in(0,1)$ and $t_{n}>0$ for all $n \geq 1$ and $\lim _{n \rightarrow \infty} t_{n}=\lim _{n \rightarrow \infty}\left(\frac{\alpha_{n}}{t_{n}}\right)=0$. Then $x_{n} \rightarrow z=P \circ g z$, where $P$ is the sunny nonexpansive retraction from $C$ onto $F(\mathcal{T})$.

Proof. It follows from [[18], Theorem 3.3] that $\left(E, C,\left\{S_{n}\right\},\left\{\alpha_{n}\right\}, P\right)$ has the "Browder's property", where $S_{n}=T\left(t_{n}\right)$, for any $n \geq 1$. Hence the conclusion of Theorem 5.1 can be obtained immediately from Theorem 4.3.

Theorem 5.2 Let $H$ be a real Hilbert space, $C$ be a nonempty, closed and convex subset of $H, \mathcal{T}:=\{T(t): t \geq 0\}: C \rightarrow C$ be a nonexpansive semigroup with $F(\mathcal{T}):=\bigcap_{t \geq 0} F(T(t)) \neq \emptyset$ and the mapping $t \alpha\|T(t) x-y\|^{2}$ is measurable for each $x \in C$ and $y \in H$. Let $\left\{\sigma_{n}\right\}:[0, \infty) \rightarrow[0, \infty)$ be a sequence of measurable functions such that $\int_{0}^{\infty} \sigma_{n}(t) d t=1$ for each $n \geq 1, \lim _{n \rightarrow \infty} \sigma_{n}(t)=0$ for almost every $t \geq 0$, $\lim _{n \rightarrow \infty} \int_{0}^{\infty}\left|\sigma_{n}(t+s)-\sigma_{n}(t)\right| d t=0$, for all $s \geq 0$ and there exists $\beta \in L_{\text {loc }}^{1}[0, \infty)$ such that $\sup _{n} \sigma_{n}(t) \leq \beta(t)$ for almost every $t \geq 0$, where $\beta \in L_{\text {loc }}^{1}[0, \infty)$ means a restriction of $\beta$ on $[0, s]$ belongs to $L^{1}[0, s]$ for each $s>0$. Let $g: C \rightarrow C$ be a weakly contractive mapping, $\left\{\alpha_{n}\right\}$ be a real sequence in $(0,1]$ such that $\alpha_{n} \rightarrow 0$ and let $\left\{\beta_{n}\right\}$ be a sequence in $0[1]$ such that $\beta_{n} \rightarrow 0$ and $\sum_{n=0}^{\infty} \beta_{n}=\infty$. Let $x$ and $y_{0}$ be points of $C$ and let $\left\{x_{n}\right\}$ and $\left\{y_{n}\right\}$ be the sequences which are defined by

$$
x_{n}=\alpha_{n} g\left(x_{n}\right)+\left(1-\alpha_{n}\right) \int_{0}^{\infty} \sigma_{n}(t) T(t) x_{n} d t, \quad n \geq 1,
$$

and

$$
y_{n+1}=\beta_{n} g\left(x_{n}\right)+\left(1-\beta_{n}\right) \int_{0}^{\infty} \sigma_{n}(t) T(t) y_{n} d t, \quad n \geq 1,
$$


respectively. Then both $\left\{x_{n}\right\}$ and $\left\{y_{n}\right\}$ converge strongly to $z=P \circ g z$, where $P$ is the metric projection from $C$ onto $F(\mathcal{T})$.

Proof. By the assumption that $\mathcal{T}:=\{T(t): t \geq 0\}: C \rightarrow C$ is a nonexpansive semiproup, hence for each $n \geq 1, S_{n}:=\int_{0}^{\infty} \sigma_{n}(t) T(t) d t: C \rightarrow C$ is a nonexpansive mapping. From [[19], Theorem 10], we know that $\left(H, C,\left\{S_{n}\right\},\left\{\alpha_{n}\right\}, P\right)$ and $\left(H, C,\left\{S_{n}\right\},\left\{\beta_{n}\right\}, P\right)$ have the Browder's and Halpern's property, respectively. Hence the conclusions of Theorem 5.2 follow immediately from Theorems 4.3 and 4.5 .

Definition 5.3 Let $\rho_{\mathrm{E}}:[0, \infty) \rightarrow[0, \infty)$ be the modulus of smoothness of a Banach space $E$ defined by

$$
\rho_{E}(t)=\sup \left\{\frac{1}{2}(\|x+y\|+\|x-y\|)-1: x \in S(E),\|y\| \leq t\right\}
$$

where $S(E)=\{x \in E:\|x\|=1\}$.

A Banach space $E$ is said to be uniformly smooth if $\frac{\rho_{E}(t)}{t} \rightarrow 0$ as $t \rightarrow 0$. Let $q>1$.

A Banach space $E$ is said to be q-uniformly smooth, if there exists a fixed constant $c>0$ such that $\rho_{E}(t) \leq c t^{q}$. It is well-known that $E$ is uniformly smooth if and only if the norm of $E$ is uniformly Fréchet differentiable. If $E$ is $q$-uniformly smooth, then $q \leq 2$ and $E$ is uniformly smooth.

Lemma 5.4 (Xu [20]) Let $E$ be a real 2-uniformly smooth Banach space with the best smooth constant $K$. Then the following inequality holds:

$$
\|x+y\|^{2} \leq\|x\|^{2}+2\langle y, J(x)\rangle+2\|K y\|^{2},
$$

for any $x, y E$, where $K$ is a positive constant. The minimal positive constant $K$ satisfying the above inequality is said to be the best smooth constant.

\subsection{Application to a system of variational inclusions}

Theorem 5.5 Let $E$ be a uniformly convex and 2-uniformly smooth Banach space with the best smooth constant $K$. Let $M_{i}: E \rightarrow 2^{E}$ be maximal monotone mappings and let $A_{i}: E \rightarrow E$ be $\gamma_{i}$-inverse-strongly accretive mappings, $i=1,2$. Let $T: E \rightarrow E$ be a $\lambda$-strict pseudocontraction with a fixed point. Define a mapping $S$ by $S x=\left(1-\frac{\lambda}{K^{2}}\right) x+\frac{\lambda}{K^{2}} T x$, for all $x \in E$. Let $g: E \rightarrow E$ be a $\psi$-weak concraction. Assume that $\Omega=F(T) \cap F(Q) \neq$ $\varnothing$, where $Q: E \rightarrow E$ is a mapping which is defined by

$$
Q(x)=J_{\left(M_{1}, \rho_{1}\right)}\left(I-\rho_{1} A_{1}\right) J_{\left(M_{2}, \rho_{2}\right)}\left(I-\rho_{2} A_{2}\right) x, \quad x \in E ，
$$

and $J_{\left(M_{i}, \rho_{i}\right)}: E \rightarrow E$ is the resolvent operator associated with $M_{i}$ defined by $J_{\left(M_{i}, \rho_{i}\right)}(x)=\left(I+\rho_{i} M\right)^{-1}(x), x \in E$, where $\rho$ is any positive number and $I$ is the identity mapping. Let $x_{1}=u$ and $\left\{x_{n}\right\}$ be a sequence defined by

$$
x_{n+1}=\alpha_{n} g\left(x_{n}\right)+\beta_{n} x_{n}+\left(1-\beta_{n}-\alpha_{n}\right)\left[\mu S x_{n}+(1-\mu) Q x_{n}\right], \quad \forall n \geq 1,
$$

where $\mu \in(0,1), \rho_{i} \in\left(0, \frac{\gamma_{i}}{K^{2}}\right), i=1,2$, and $\left\{\alpha_{n}\right\}$ and $\left\{\beta_{n}\right\}$ are sequences in $(0,1)$. If the following conditions are satisfied:

(i) $0<\lim \inf _{n \rightarrow \infty} \beta_{n} \leq \lim \sup _{n \rightarrow \infty} \beta_{n}<1$;

(ii) $\lim _{n \rightarrow \infty} \alpha_{n}=0$ and $\sum_{n=1}^{\infty} \alpha_{n}=\infty$, 
then $\left\{x_{n}\right\}$ converges strongly to $x^{*}=P_{\Omega} g\left(x^{*}\right)$, where $P_{\Omega}$ is the sunny nonexpansive retraction from $E$ onto $\Omega$ and $\left(x^{*}, y^{*}\right)$, where $y^{*}=J_{\left(M_{2}, \rho_{2}\right)}\left(x^{*}-\rho_{2} A_{2} x^{*}\right)$ is a solution of the following system of quasivariational inclusions:

$$
\left\{\begin{array}{l}
0 \in x^{*}-\gamma^{*}+\rho_{1}\left(A_{1} y^{*}+M_{1} x^{*}\right), \\
0 \in \gamma^{*}-x^{*}+\rho_{2}\left(A_{2} x^{*}+M_{2} y^{*}\right) .
\end{array}\right.
$$

Proof. By the assumptions, we know that the mappings $S$ and $Q$ are nonexpansive on $E$. From [21], the mapping $U:=\mu S+(1-\mu) Q$ is also nonexpansive on $E$. Since

$$
\beta_{n} x_{n}+\left(1-\beta_{n}-\alpha_{n}\right)\left[\mu S x_{n}+(1-\mu) Q x_{n}\right]=\left(1-\alpha_{n}\right)\left(\frac{\beta_{n} I}{1-\alpha_{n}}+\left(1-\frac{\beta_{n}}{1-\alpha_{n}}\right) U\right)\left(x_{n}\right),
$$

where $\frac{\beta_{n} I}{1-\alpha_{n}}+\left(1-\frac{\beta_{n}}{1-\alpha_{n}}\right) U$ is a nonexpansive on $C$ (see [21]), hence (4.4) can be rewritten as follows:

$$
x_{n+1}=\alpha_{n} g\left(x_{n}\right)+\left(1-\alpha_{n}\right)\left(\frac{\beta_{n} I}{1-\alpha_{n}}+\left(1-\frac{\beta_{n}}{1-\alpha_{n}}\right) U\right)\left(x_{n}\right), \quad \forall n \geq 1 .
$$

From [[22], Theorem 2.1], $\left.\left(E, C,\left\{S_{n}\right\},\left\{\alpha_{n}\right\}\right), P_{\Omega}\right)$ has the "Halpern's property", where

$$
S_{n}=\frac{\beta_{n} I}{1-\alpha_{n}}+\left(1-\frac{\beta_{n}}{1-\alpha_{n}}\right) U, \quad n \geq 1
$$

Hence for any $u \in E$ the sequence $\left\{y_{n}\right\}$ defined by $y_{1}=u \in E$ and

$$
y_{n+1}=\alpha_{n} u+\left(1-\alpha_{n}\right) S_{n} y_{n}, \quad \forall n \geq 1,
$$

converges strongly to $x^{*}=P_{\Omega} u$ and $\left(x^{*}, y^{*}\right)$, where $y^{*}=J_{M_{2}, \rho_{2}}\left(I-\rho_{2} A_{2}\right) x^{*}$, is a solution to the system of quasivariational inclusions (5.5). Hence from Theorem 4.3, we know that $x_{n} \rightarrow x^{*}=P_{\Omega} g\left(x^{*}\right)$, and $\left(x^{*}, y^{*}\right)$, where $y^{*}=J_{M_{2}, \rho_{2}}\left(I-\rho_{2} A_{2}\right) x^{*}$, is a solution to the system of quasivariational inclusions (5.5).

This completes the proof of Theorem 5.5.

\subsection{Application to equilibrium problem}

Let $H$ be a real Hilbert space, $C$ be a nonempty, closed and convex subset of $H$ and let $G: C \times C \rightarrow \mathcal{R}$ be an equilibrium function, i.e., $G(u, u)=0$, for any $u \in C$. The equilibrium problem is to find $x^{*} \in C$ such that $G\left(x^{*}, y\right) \geq 0$, for all $y \in C$. The solution set of equilibrium problem is denoted by $E P(G)$.

For solving equilibrium problem of a function $G: C \times C \rightarrow \mathcal{R}$, let us assume that $G$ satisfies the following conditions:

(A1) $G(x, x)=0$;

(A2) $G(x, y)+G(y, x) \leq 0, \forall x, y \in C$;

(A3) for each $x, y, z \in C$, lim $\sup _{t \rightarrow 0} G(t z+(1-t) x, y) \leq G(x, y)$;

(A4) for each $x \in C, y \mapsto G(x, y)$ is convex and lower semi-continuous.

Let $\left\{T_{i}\right\}$ be an infinite family of nonexpansive mappings on $C$ and let $\left\{\lambda_{i}\right\}$ be a real sequence in 0[1]. Following Shimoji and Takahashi [23], for any $n \geq 1$, we define a mapping 


$$
\begin{aligned}
& W_{n}: C \rightarrow C \text { by } \\
& \left\{\begin{array}{l}
U_{n, n+1}=I, \\
U_{n, n}=\lambda_{n} T_{n} U_{n, n+1}+\left(1-\lambda_{n}\right) I, \\
\vdots \\
U_{n, k}=\lambda_{k} T_{k} U_{n, k+1}+\left(1-\lambda_{k}\right) I, \\
\vdots \\
U_{n, 2}=\lambda_{2} T_{2} U_{n, 3}+\left(1-\lambda_{2}\right) I, \\
W_{n}=U_{n, 1}=\lambda_{1} T_{1} U_{n, 2}+\left(1-\lambda_{1}\right) I .
\end{array}\right.
\end{aligned}
$$

Lemma 5.6 (Combettes and Hirstoaga [24]) Let $G: C \times C \rightarrow \mathcal{R}$ be an equilibrium function which satisfies the conditions (A1)-(A4). For $r>0$ and $x \in H$ define the mapping $S_{r}: H \rightarrow C$ by

$$
S_{r}(x)=\left\{z \in C: G(z, y)+\frac{1}{r}\langle y-z, z-x\rangle \geq 0, \quad \forall y \in C\right\},
$$

then $S_{r}$ is well defined and the following hold: (1) $S_{r}$ is single-valued; (2) $S_{r}$ is firmly nonexpansive, i.e., $\left\|S_{r} x-S_{r}\right\|^{2} \leq\left\langle S_{r} x-S_{r}, x-y\right\rangle$, for all $x, y \in H$; (3) $F\left(S_{r}\right)=\operatorname{EP}(G)$; (4) $\operatorname{EP}(G)$ is closed and convex.

Theorem 5.7 Let $H$ be a real Hilbert space, $\left\{T_{i}\right\}: H \rightarrow H$ be an infinite family of nonexpansive mappings, $G_{k}: H \times H \rightarrow R, k=1,2, \ldots, N$ be functions which satisfy conditions (A1)-(A4) such that $\mathcal{F}:=\left(\cap_{k=1}^{N} \operatorname{EP}\left(G_{k}\right) \cap \cap_{n=1}^{\infty} F\left(T_{n}\right)\right) \neq \emptyset, A: H \rightarrow H$ be a strongly positive bounded linear operator with coefficient $\bar{\gamma}>0$ and $\Phi: H \rightarrow H$ be a $\psi$-weak contraction. Moreover, let $\left\{r_{k, n}\right\}_{k=1}^{N}$ be positive sequences, $\left\{\alpha_{n}\right\}$ be a sequence in $0[1]$, and $\gamma$ be a real number such that $0<\gamma<\bar{\gamma}$. Assume that

(i) $\lim _{n \rightarrow \infty} \alpha_{n}=0$;

(ii) $\lim _{n \rightarrow \infty} r_{k, n}=\hat{r}_{k}$ for each $k=1,2, \ldots, N$.

Let $\left\{x_{n}\right\}$ be the sequence defined by

$$
x_{n}=\alpha_{n} \gamma \Phi\left(x_{n}\right)+\left(I-\alpha_{n} A\right) W_{n} S_{r_{1}, n}^{1} S_{r_{2}, n}^{2} \ldots S_{r_{N}, n}^{N} x_{n}, \quad n \geq 1,
$$

where $W_{n}$ is the mapping generated by $\left\{T_{i}\right\}$ and $\left\{\lambda_{n}\right\}$ as in (5.7), and for each $k=1$, $2, \ldots, N$ and each $n \geq 1, S_{r_{k}, n}^{k}$ is the mapping generated by $G_{k}$ and $r_{k, n}$ as in Lemma 5.6. Then $\left\{x_{n}\right\}$ converges strongly $x^{*}=P_{\mathcal{F}}(I-(A-\gamma \Phi)) x^{*}$.

Proof. Since for each $n \geq 1$ and for each $k=1,2, \ldots, N$ the mappings $W_{n}$ and $S_{r_{k}, n}^{k}$ all are nonexpansive on $H$. Define a mapping $V_{n}: H \rightarrow H$ by:

$$
V_{n} x:=\alpha_{n} \gamma \Phi(x)+\left(I-\alpha_{n} A\right) W_{n} S_{r_{1}, n}^{1} S_{r_{2}, n}^{2} \ldots S_{r_{N}, n}^{N} x
$$

It is easy to see that for each $n \geq 1, V_{n}$ is a $\psi$-weakly contractive mapping. Hence there exists a unique $x_{n} \in H$ such that $x_{n}=V_{n} x_{n}$. This shows that the sequence $\left\{x_{n}\right\}$ defined by (5.8) is well-defined. By the similar method as in [[25], Theorem 8], we can prove that $x_{n} \rightarrow x^{*}$, where $x^{*}$ is the unique solution of the variational inequality

$$
\left\langle(A-\gamma \Phi) x^{*}, x-x^{*}\right\rangle \geq 0, \quad \forall x \in \mathcal{F},
$$


that is $x^{*}=P_{\mathcal{F}}(I-(A-\gamma \Phi)) x^{*}$, or, equivalently $x^{*}$ is the unique solution of the minimization problem:

$$
\min _{x \in \mathcal{F}} \frac{1}{2}\langle A x, x\rangle+h(x)
$$

where $h$ is the potential function for $\gamma \Phi$.

\section{Acknowledgements}

The authors would like to express their thanks to the referees for their helpful comments and suggestions. This work was supported by the National Research Foundation of Korean Grant funded by the Korean Government (20110002581).

\section{Author details}

${ }^{1}$ College of Statistics and Mathematics, Yunnan University of Finance and Economics, Kunming, Yunnan 650221, China ${ }^{2}$ Department of Mathematics Education, Kyungnam University, Masan, Kyungnam 631-701, South Korea ${ }^{3}$ Department of Applied Mathematics, The Hong Kong Polytechnic University, Hung Hom, Kowloon, Hong kong

\section{Authors' contributions}

S-sC and JKK conceived the study and participated in its design and coordination. JKK and HWJL suggested many good ideas that are useful for achievement this paper and made the revision. JKK and CKC prepared the manuscript initially and performed all the steps of proof in this research. All authors read and approved the final manuscript.

\section{Competing interests}

The authors declare that they have no competing interests.

Received: 4 September 2011 Accepted: 3 April 2012 Published: 3 April 2012

\section{References}

1. Alber, Yal, Guerre-Delabriere, S: Principles of weakly contractive maps in Hilbert spaces. In: Gohberg I, Lyubich Yu (eds.) New Results in Operator Theory, vol. 98, pp. 7-22. Advances and Appl. Birkhauser, Basel (1997)

2. Rhoades, BE: Some theorems on weakly contractive maps. Nonlinear Anal. 47, 2683-2693 (2001)

3. Göhde, D: Zum Prinzip der kontraktiven Abbildung. Math Nachr. 30, 251-258 (1965)

4. Browder, FE: Convergence of approximations to fixed points of nonexpansive nonlinear mappings in Banach space. Arch Ration Mech Anal. 24, 82-90 (1967)

5. Halpern, B: Fixed points of nonexpansive maps. Bull Am Math Soc. 73, 957-961 (2004)

6. Reich, S: Strong convergence theorems for resolvents of accretive operators in Banach spaces. J Math Anal Appl. 75, 287-292 (1980)

7. Xu, HK: Viscosity approximation methods for nonexpansive mappings. J Math Anal Appl. 298, 279-291 (2004)

8. Xu, HK: Another control condition in an iterative method for nonexpansive mappings. Bull Aust Math Soc. 65, 109-113 (2002)

9. Xu, HK: Iterative algorithms for nonlinear operators. J Lond Math Soc. 66, 240-252 (2002)

10. Moudafi, A: Viscosity approximation methods for fixed point problems. J Math Anal Appl. 241, 46-55 (2000)

11. Suzuki, T: Moudafi's viscosity approximations with Meir-Keeler contractions. J Math Anal Appl. 325, 342-352 (2007)

12. Song, YS, Chen, R: Iterative approximation to common fixed points ofnonexpansive mapping sequences in Banach spaces. Nonlinear Anal. 66, 591-603 (2007)

13. Song, YS, Chen, R: Viscosity approximation methods for nonexpansive mappings in Banach spaces. Nonlinear Anal. 66, 1016-1024 (2007)

14. Song, YS: Equivalent theorems of the convergence between proximal type algorithms. Nonlinear Anal. 71, 293-300 (2009)

15. Petrusel, A, Yao, JC: Viscosity approximation to common fixed points of families of nonexpansive mappings with generalized contractions mappings. Nonlinear Anal. 69, 1100-1111 (2008)

16. Meir, A, Keeler, E: A theorem on contraction mappings. J Math Anal Appl. 28, 326-329 (1969)

17. Reich, S: Asymptotic behavior of contractions in Banach spaces. J Math Anal Appl. 44, 57-70 (1973)

18. $\mathrm{Xu}, \mathrm{HK}$ : A strong convergence theorem for contraction semigroups in Banach spaces. Bull Aust Math Soc. 72, 371-379 (2005)

19. Shioji, N, Takahashi, W: Strong convergence theorems for asymptotically nonexpansive semigroups in Hilbert spaces. Nonlinear Anal. 34, 87-99 (1998)

20. Xu, HK: Inequalities in Banach spaces with applications. Nonlinear Anal. 16, 1127-1138 (1991)

21. Bruck, RE Jr: Properties of fixed point sets of nonexpansive mappings in Banach spaces. Trans Am Math Soc. 179, 251-262 (1973)

22. Qin, X, Chang, SS, Cho, YJ, Kang, SM: Approximation of solutions to system of variational inclusions in Banach spaces. J Inequal Appl 2010 (2010). 16 (Article ID 916806). doi:10.1155/2010/916806

23. Shimoji, K, Takahashi, W: Strong convergence to common fixed points of infinite non-expansive mappings and applications. Taiwanese J Math. 5, 387-404 (2001)

24. Combettes, PL, Hirstoaga, SA: Equilibrium programming in Hilbert spaces. J Nonlinear Convex Anal. 6(1)117-136 (2005) 
25. Colao, V, Acedo, GL, Marino, G: An implicit method for finding common solutions of variational inequalities and systems of equilibrium problems and fixed points of infinite family of nonexpansive mappings. Nonlinear Anal. 71, 2708-2715 (2009)

doi:10.1186/1687-1812-2012-55

Cite this article as: Chang et al:: On the equivalence between some kinds of implicit and explicit iterations with applications. Fixed Point Theory and Applications 2012 2012:55.

Submit your manuscript to a SpringerOpen ${ }^{\odot}$ journal and benefit from:

- Convenient online submission

- Rigorous peer review

- Immediate publication on acceptance

- Open access: articles freely available online

- High visibility within the field

- Retaining the copyright to your article

Submit your next manuscript at $\boldsymbol{\nabla}$ springeropen.com 